GSA Data Repository Item 2011273

\title{
Large-scale silicate liquid immiscibility during differentiation of tholeiitic basalt to granite and the origin of the Daly gap
}

Bernard Charlier, Olivier Namur, Michael J. Toplis, Pierre Schiano, Nicolas Cluzel, Michael D. Higgins, Jacqueline Vander Auwera

\section{Materials and Methods}

Drill-cores of $47.6 \mathrm{~mm}$ diameter were drilled in the Critical Zone of the Sept Iles intrusion in the period 1995-1996, perpendicular to the layering with an azimuth of $325^{\circ}$ and an inclination of $65^{\circ}$. Cores were split and individual samples represent 0.32-13.2 $\mathrm{m}$ long portions (2.4 $\mathrm{m}$ on average). Phosphorous was measured in 895 samples by ALS ChemexChimitec (Val-d'Or, Canada) using the gravimetric method by precipitation of magnesium ammonium phosphate hexahydrate. Density measurements were performed on 228 selected rocks by the water-immersion method. Plagioclase composition was determined by XRF on Li-borate fused glass on mineral separates (60-150 $\mu \mathrm{m})$ performed using flotation in bromoform and a Frantz isodynamic magnetic separator. Final purification was carried out by $\mathrm{HCl}$ leaching to dissolve any apatite grains and by hand-picking under the microscope. The composition of olivine, clinopyroxene and melt inclusions were determined with a Cameca SX100 electron microprobe at Clermont-Ferrand. For olivine and clinopyroxene, beam conditions of $15 \mathrm{kV}$ and $15 \mathrm{nA}$ were used, counting times were $10 \mathrm{~s}$ and the electron beam was focused. For melt inclusions analyses, the beam was defocused to $10 \mu \mathrm{m}$ and the current was $8 \mathrm{nA}$. The following standards were used: wollastonite for $\mathrm{Si}$ and $\mathrm{Ca}, \mathrm{Al}_{2} \mathrm{O}_{3}$ for $\mathrm{Al}$, fayalite for $\mathrm{Fe}$, forsterite for $\mathrm{Mg}, \mathrm{MnTiO}_{3}$ for $\mathrm{Ti}$ and $\mathrm{Mn}$, albite for $\mathrm{Na}$, orthose for $\mathrm{K}$ and apatite for P. Prior to analyses, finely crystallized melt inclusions in apatite were rehomogenised using heating stage experiments under air conditions and in a vertical dropquench gas mixing furnace with oxygen fugacity control at FMQ-1 using $\mathrm{N}_{2}-\mathrm{CO}_{2}$ gas mixtures. Apatite grains were loaded in a small $\mathrm{Pt}$ crucible in the hotspot of the furnace and heated from $25^{\circ} \mathrm{C}$ to $1080-1100^{\circ} \mathrm{C}$ in 2 hours. The experiment was held at the final temperature for 30 min to allow equilibration of the melts with their host apatite before dropquenching into water. 
Table DR1. Composition of olivine in the Critical Zone of the Sept Iles layered intrusion

\begin{tabular}{|c|c|c|c|c|c|c|c|c|c|c|c|}
\hline & $\begin{array}{c}\text { Depth } \\
\text { (m) }\end{array}$ & $\mathbf{n}$ & $\mathrm{SiO}_{2}$ & $\mathrm{TiO}_{2}$ & $\mathrm{Al}_{2} \mathrm{O}_{3}$ & $\mathrm{FeO}$ & MnO & MgO & $\mathrm{CaO}$ & Total & $\begin{array}{c}\text { Fo } \\
\text { (mol\%) }\end{array}$ \\
\hline ON-07-120 & 226 & 13 & 31.30 & 0.02 & 0.01 & 58.01 & 1.40 & 8.72 & 0.06 & 99.52 & 21.1 \\
\hline ON-07-125 & 206 & 16 & 32.48 & 0.03 & 0.01 & 53.83 & 1.36 & 12.83 & 0.04 & 100.58 & 29.8 \\
\hline 33.3 & 192.8 & 11 & 33.35 & 0.02 & 0.01 & 49.26 & 1.33 & 16.40 & 0.12 & 100.49 & 37.2 \\
\hline 49.6 & 176.5 & 10 & 33.37 & 0.03 & 0.01 & 48.23 & 1.19 & 17.30 & 0.14 & 100.27 & 39.0 \\
\hline 63.9 & 162.2 & 12 & 33.34 & 0.01 & 0.01 & 48.10 & 1.34 & 17.44 & 0.07 & 100.31 & 39.3 \\
\hline 70.6 & 155.5 & 12 & 33.81 & 0.02 & 0.01 & 46.68 & 1.19 & 18.69 & 0.10 & 100.50 & 41.6 \\
\hline 82.1 & 144.0 & 13 & 34.42 & 0.03 & 0.00 & 44.45 & 1.03 & 20.56 & 0.10 & 100.59 & 45.2 \\
\hline 88.1 & 138.0 & 17 & 34.42 & 0.01 & 0.01 & 42.57 & 0.59 & 22.23 & 0.04 & 99.87 & 48.2 \\
\hline 93.6 & 132.5 & 15 & 34.28 & 0.01 & 0.01 & 43.81 & 0.94 & 21.14 & 0.06 & 100.25 & 46.2 \\
\hline 100.8 & 125.3 & 18 & 34.77 & 0.01 & 0.01 & 42.87 & 0.91 & 22.18 & 0.05 & 100.80 & 48.0 \\
\hline 110.7 & 115.4 & 23 & 34.83 & 0.02 & 0.01 & 43.00 & 0.94 & 22.15 & 0.06 & 101.01 & 47.9 \\
\hline 118.8 & 107.3 & 14 & 35.14 & 0.01 & 0.01 & 38.59 & 0.79 & 24.82 & 0.04 & 99.40 & 53.4 \\
\hline 132.2 & 93.9 & 12 & 34.98 & 0.02 & 0.01 & 39.85 & 0.81 & 24.33 & 0.06 & 100.06 & 52.1 \\
\hline 135.9 & 90.2 & 6 & 35.43 & 0.01 & 0.01 & 40.02 & 0.78 & 24.87 & 0.06 & 101.18 & 52.5 \\
\hline 148 & 78.1 & 12 & 35.66 & 0.02 & 0.01 & 35.82 & 0.62 & 28.03 & 0.05 & 100.21 & 58.2 \\
\hline 152.4 & 73.7 & 14 & 35.45 & 0.01 & 0.01 & 36.03 & 0.69 & 27.76 & 0.05 & 100.00 & 57.9 \\
\hline 173.8 & 52.3 & 9 & 35.85 & 0.02 & 0.01 & 37.35 & 0.59 & 26.80 & 0.03 & 100.66 & 56.1 \\
\hline 179.8 & 46.3 & 15 & 34.98 & 0.02 & 0.01 & 38.05 & 0.77 & 25.72 & 0.06 & 99.61 & 54.6 \\
\hline 190.9 & 35.2 & 15 & 35.64 & 0.02 & 0.01 & 37.20 & 0.76 & 26.57 & 0.06 & 100.26 & 56.0 \\
\hline 200.9 & 25.2 & 9 & 35.73 & 0.02 & 0.02 & 38.39 & 0.66 & 25.83 & 0.05 & 100.70 & 54.5 \\
\hline 214.8 & 11.3 & 13 & 35.99 & 0.03 & 0.02 & 33.89 & 0.68 & 29.35 & 0.09 & 100.05 & 60.7 \\
\hline 218.8 & 7.3 & 17 & 35.85 & 0.03 & 0.01 & 34.80 & 0.61 & 28.72 & 0.03 & 100.05 & 59.5 \\
\hline
\end{tabular}

$\mathrm{n}$ is the number of microprobe analyses 
Table DR2. Composition of clinopyroxene in the Critical Zone of the Sept Iles layered intrusion

\begin{tabular}{|c|c|c|c|c|c|c|c|c|c|c|c|c|}
\hline Sample & $\begin{array}{c}\text { Depth } \\
\text { (m) }\end{array}$ & $\mathbf{n}$ & $\mathrm{SiO}_{2}$ & $\mathrm{TiO}_{2}$ & $\mathrm{Al}_{2} \mathrm{O}_{3}$ & $\mathrm{FeO}$ & MnO & MgO & $\mathrm{CaO}$ & $\mathrm{Na}_{2} \mathrm{O}$ & Total & Mg\# \\
\hline $07-120$ & 226 & 16 & 51.37 & 0.31 & 1.09 & 15.64 & 0.60 & 10.82 & 20.18 & 0.24 & 100.24 & 55.2 \\
\hline $07-125$ & 206 & 10 & 51.94 & 0.30 & 0.98 & 13.81 & 0.53 & 12.11 & 20.72 & 0.27 & 100.66 & 61.0 \\
\hline 33.3 & 192.8 & 12 & 51.10 & 0.57 & 1.44 & 14.27 & 0.61 & 11.39 & 20.01 & 0.32 & 99.71 & 58.7 \\
\hline 49.6 & 176.5 & 14 & 51.18 & 0.60 & 1.52 & 13.81 & 0.60 & 11.63 & 20.06 & 0.34 & 99.75 & 60.0 \\
\hline 63.9 & 162.2 & 16 & 51.10 & 0.44 & 1.35 & 13.56 & 0.68 & 11.86 & 20.33 & 0.23 & 99.55 & 60.9 \\
\hline 70.6 & 155.5 & 10 & 51.45 & 0.56 & 1.55 & 13.27 & 0.60 & 12.03 & 20.17 & 0.33 & 99.96 & 61.8 \\
\hline 88.1 & 138 & 12 & 51.45 & 0.48 & 1.59 & 11.00 & 0.31 & 13.11 & 21.06 & 0.30 & 99.31 & 68.0 \\
\hline 118.3 & 107.8 & 12 & 51.70 & 0.57 & 1.72 & 10.20 & 0.41 & 13.85 & 20.94 & 0.31 & 99.70 & 70.8 \\
\hline 132.2 & 93.9 & 17 & 51.58 & 0.56 & 1.76 & 10.28 & 0.40 & 13.83 & 20.74 & 0.31 & 99.47 & 70.6 \\
\hline 148 & 78.1 & 12 & 51.72 & 0.63 & 1.76 & 9.85 & 0.33 & 14.01 & 20.92 & 0.44 & 99.66 & 71.7 \\
\hline 152.4 & 73.7 & 12 & 51.41 & 0.61 & 1.97 & 10.13 & 0.34 & 14.07 & 20.57 & 0.35 & 99.45 & 71.2 \\
\hline 169.8 & 56.3 & 10 & 51.93 & 0.65 & 1.84 & 9.73 & 0.29 & 14.27 & 21.08 & 0.35 & 100.12 & 72.3 \\
\hline 173.8 & 52.3 & 9 & 52.22 & 0.59 & 1.85 & 9.60 & 0.33 & 14.11 & 21.46 & 0.31 & 100.47 & 72.4 \\
\hline 179.8 & 46.3 & 14 & 51.72 & 0.54 & 1.72 & 10.23 & 0.38 & 13.90 & 20.86 & 0.33 & 99.68 & 70.8 \\
\hline 190.9 & 35.2 & 12 & 51.74 & 0.63 & 1.92 & 9.98 & 0.37 & 14.02 & 20.77 & 0.33 & 99.77 & 71.5 \\
\hline 214.8 & 11.3 & 10 & 51.61 & 0.64 & 1.98 & 9.34 & 0.36 & 14.15 & 20.83 & 0.43 & 99.35 & 73.0 \\
\hline 218.8 & 7.3 & 6 & 51.57 & 0.53 & 1.70 & 10.79 & 0.40 & 13.97 & 20.19 & 0.31 & 99.46 & 69.8 \\
\hline 221.6 & 4.5 & 16 & 52.10 & 0.62 & 1.99 & 9.74 & 0.37 & 14.15 & 20.94 & 0.41 & 100.32 & 72.1 \\
\hline
\end{tabular}

$\mathrm{n}$ is the number of microprobe analyses 
Table DR3. Composition of plagioclase in the Critical Zone of the Sept Iles layered intrusion

\begin{tabular}{|l|c|c|c|c|c|c|c|c|c|c|}
\hline Samples & $\begin{array}{c}\mathbf{D e p t h} \\
\mathbf{( m )}\end{array}$ & $\mathbf{S i O}_{\mathbf{2}}$ & $\mathbf{T i O}_{\mathbf{2}}$ & $\mathbf{A l}_{\mathbf{2}} \mathbf{O}_{\mathbf{3}}$ & $\mathbf{F e}_{\mathbf{2}} \mathbf{O}_{\mathbf{3}}$ & $\mathbf{C a O}$ & $\mathbf{N a}_{\mathbf{2}} \mathbf{O}$ & $\mathbf{K}_{\mathbf{2}} \mathbf{O}$ & $\mathbf{T o t a l}$ & $\begin{array}{c}\mathbf{A n} \\
(\mathbf{m o l} \mathbf{)})\end{array}$ \\
\hline ON07-120 & 226 & 59.80 & 0.03 & 25.23 & 0.23 & 7.05 & 7.45 & 0.19 & 99.98 & 34.3 \\
\hline ON07-125 & 206 & 60.72 & 0.08 & 24.94 & 0.19 & 6.99 & 7.09 & 0.41 & 100.42 & 35.3 \\
\hline 33.3 & 192.8 & 57.37 & 0.06 & 25.63 & 0.42 & 8.71 & 6.02 & 0.83 & 99.03 & 44.4 \\
\hline 49.6 & 176.5 & 57.64 & 0.06 & 25.83 & 0.43 & 8.86 & 6.08 & 0.84 & 99.74 & 44.6 \\
\hline 63.9 & 162.2 & 57.79 & 0.06 & 25.30 & 0.33 & 8.44 & 6.12 & 0.90 & 98.94 & 43.2 \\
\hline 70.6 & 155.5 & 57.57 & 0.06 & 25.91 & 0.42 & 8.74 & 6.08 & 0.85 & 99.63 & 44.3 \\
\hline 82.1 & 144.0 & 57.20 & 0.06 & 25.91 & 0.40 & 8.90 & 5.94 & 0.79 & 99.20 & 45.3 \\
\hline 88.1 & 138.0 & 56.74 & 0.07 & 26.36 & 0.39 & 9.53 & 5.84 & 0.64 & 99.57 & 47.4 \\
\hline 93.6 & 132.5 & 57.28 & 0.07 & 25.92 & 0.39 & 9.00 & 6.14 & 0.56 & 99.36 & 44.8 \\
\hline 100.8 & 125.3 & 57.36 & 0.07 & 25.97 & 0.40 & 8.93 & 6.23 & 0.55 & 99.51 & 44.2 \\
\hline 118.3 & 107.8 & 56.31 & 0.06 & 26.78 & 0.39 & 9.99 & 5.61 & 0.59 & 99.73 & 49.6 \\
\hline 132.2 & 93.9 & 56.08 & 0.06 & 26.99 & 0.39 & 10.07 & 5.61 & 0.47 & 99.65 & 49.8 \\
\hline 135.9 & 90.2 & 56.05 & 0.07 & 26.87 & 0.41 & 9.98 & 5.64 & 0.57 & 99.59 & 49.4 \\
\hline 148.0 & 78.1 & 56.08 & 0.07 & 26.86 & 0.42 & 10.14 & 5.63 & 0.43 & 99.63 & 49.9 \\
\hline 152.4 & 73.7 & 54.69 & 0.06 & 27.70 & 0.45 & 11.14 & 5.07 & 0.46 & 99.57 & 54.8 \\
\hline 169.2 & 56.9 & 55.15 & 0.07 & 27.49 & 0.38 & 10.97 & 5.29 & 0.42 & 99.77 & 53.4 \\
\hline 173.8 & 52.3 & 55.05 & 0.07 & 27.48 & 0.39 & 10.91 & 5.29 & 0.42 & 99.61 & 53.3 \\
\hline 179.8 & 46.3 & 54.54 & 0.07 & 27.44 & 0.41 & 11.07 & 5.14 & 0.33 & 98.98 & 54.3 \\
\hline 190.9 & 35.2 & 55.52 & 0.07 & 27.09 & 0.39 & 10.45 & 5.47 & 0.49 & 99.48 & 51.4 \\
\hline 192.0 & 34.1 & 55.24 & 0.07 & 26.99 & 0.36 & 10.36 & 5.39 & 0.47 & 98.88 & 51.5 \\
\hline 214.8 & 11.3 & 54.58 & 0.07 & 27.26 & 0.36 & 10.70 & 5.34 & 0.21 & 98.52 & 52.5 \\
\hline 218.8 & 7.3 & 55.54 & 0.07 & 26.88 & 0.39 & 10.41 & 5.52 & 0.31 & 99.12 & 51.0 \\
\hline 221.6 & 4.5 & 54.91 & 0.08 & 27.09 & 0.33 & 10.69 & 5.32 & 0.32 & 98.74 & 52.6 \\
\hline 223.6 & 2.5 & 55.64 & 0.07 & 27.23 & 0.39 & 10.76 & 5.52 & 0.29 & 99.90 & 51.9 \\
\hline 225.2 & 0.9 & 54.78 & 0.11 & 27.41 & 0.50 & 10.81 & 5.42 & 0.10 & 99.13 & 52.4 \\
\hline & & & & & & & & & \\
\hline
\end{tabular}


Table DR4. Composition of melt inclusions in apatite from sample at $7.3 \mathrm{~m}$

\begin{tabular}{|c|c|c|c|c|c|c|c|c|c|c|c|c|}
\hline & $\mathbf{n}$ & $\mathrm{SiO}_{2}$ & $\mathrm{TiO}_{2}$ & $\mathrm{Al}_{2} \mathrm{O}_{3}$ & $\mathrm{FeO}$ & MnO & MgO & $\mathrm{CaO}$ & $\mathrm{Na}_{2} \mathrm{O}$ & $\mathrm{K}_{2} \mathrm{O}$ & $\mathrm{P}_{2} \mathrm{O}_{5}$ & Total \\
\hline \multicolumn{13}{|l|}{ Si-Pole } \\
\hline MI44/45 & 2 & 70.43 & 0.11 & 16.15 & 0.93 & 0.09 & 0.23 & 1.69 & 4.58 & 4.61 & 0.11 & 98.93 \\
\hline MI46 & 1 & 70.25 & 0.40 & 16.22 & 1.38 & 0.11 & 0.19 & 2.02 & 4.17 & 4.84 & 0.17 & 99.75 \\
\hline MI48 & 1 & 70.17 & 0.22 & 16.61 & 0.74 & 0.11 & 0.24 & 2.09 & 3.50 & 5.44 & 0.21 & 99.32 \\
\hline MI49 & 1 & 69.99 & 0.30 & 16.45 & 1.37 & 0.17 & 0.50 & 1.85 & 4.53 & 4.71 & 0.18 & 100.06 \\
\hline MI50 & 1 & 70.67 & 0.58 & 15.07 & 2.15 & 0.08 & 0.30 & 2.33 & 4.47 & 4.49 & 0.18 & 100.31 \\
\hline MI51 & 1 & 68.55 & 0.39 & 16.14 & 2.38 & 0.11 & 0.81 & 2.47 & 3.92 & 4.28 & 0.40 & 99.45 \\
\hline MI52/53 & 2 & 70.49 & 0.37 & 16.13 & 1.27 & 0.07 & 0.39 & 1.42 & 4.78 & 4.78 & 0.19 & 99.90 \\
\hline MI55 & 1 & 69.83 & 0.20 & 15.91 & 1.70 & 0.11 & 0.62 & 1.41 & 4.67 & 5.34 & 0.25 & 100.05 \\
\hline MI56 & 1 & 69.60 & 0.27 & 16.32 & 1.48 & 0.11 & 0.66 & 1.81 & 4.24 & 5.17 & 0.21 & 99.87 \\
\hline $\mathrm{MI} / 4$ & 2 & 66.93 & 2.62 & 15.46 & 2.17 & 0.09 & 0.14 & 2.16 & 4.80 & 4.13 & 0.17 & 98.68 \\
\hline MI7/8 & 1 & 67.86 & 1.80 & 16.48 & 1.27 & 0.07 & 0.19 & 2.37 & 5.32 & 3.71 & 0.24 & 99.32 \\
\hline MI17/18 & 2 & 70.23 & 2.01 & 14.76 & 1.07 & 0.05 & 0.11 & 1.99 & 4.44 & 4.20 & 0.13 & 98.99 \\
\hline MI21/22 & 2 & 69.34 & 0.73 & 16.05 & 2.00 & 0.10 & 0.55 & 2.10 & 4.57 & 4.08 & 0.28 & 99.80 \\
\hline MI23/24/25 & 3 & 7.96 & 0.35 & 14.49 & 3.25 & 0.08 & 0.58 & 1.54 & .31 & 5.78 & 0.11 & 98.46 \\
\hline MI39 & 1 & 69.28 & 0.25 & 16.67 & 1.28 & 0.12 & 0.43 & 1.94 & 4.66 & 4.28 & 0.16 & 99.08 \\
\hline MI40/41 & 2 & 69.26 & 0.62 & 16.22 & 1.71 & 0.07 & 0.39 & 1.65 & 5.12 & 3.64 & 0.36 & 99.04 \\
\hline $\mathrm{MI} 3 / 4$ & 2 & 0.50 & 0.14 & 16.84 & 0.54 & 0.08 & 0.27 & 2.06 & 88 & 4.44 & 0.23 & 98.98 \\
\hline MI5 & 1 & 69.80 & 0.30 & 16.43 & 1.40 & 0.17 & 0.53 & 1.82 & 4.18 & 4.79 & 0.33 & 99.74 \\
\hline MI10 & 1 & 69.29 & 0.08 & 16.67 & 1.30 & 0.11 & 0.48 & 1.75 & 3.27 & 6.77 & 0.35 & 100.07 \\
\hline MI13/14 & 2 & 65.56 & 0.33 & 18.26 & 1.37 & 0.07 & 0.48 & 2.24 & 5.54 & 6.17 & 0.31 & 100.34 \\
\hline MI15 & 1 & 66.29 & 0.23 & 17.86 & 1.26 & 0.00 & 0.55 & 2.03 & 5.84 & 5.35 & 0.16 & 99.59 \\
\hline MI20/21 & 2 & 71.94 & 0.18 & 15.45 & 0.96 & 0.05 & 0.28 & 1.24 & .42 & 4.49 & 0.04 & 100.05 \\
\hline $\mathrm{MI} 22 / 23$ & 2 & 63.55 & 0.43 & 19.63 & 1.51 & 0.02 & 0.60 & 2.40 & 6.78 & 4.11 & 0.10 & 99.13 \\
\hline MI24 & 1 & 69.17 & 0.32 & 15.95 & 1.56 & 0.13 & 0.91 & 1.45 & 5.70 & 5.05 & 0.49 & 100.71 \\
\hline MI25 & 1 & 69.99 & 0.18 & 15.65 & 0.94 & 0.00 & 0.49 & 1.10 & 5.97 & 5.10 & 0.49 & 99.91 \\
\hline MI27/28 & 2 & 70.14 & 0.15 & 15.78 & 1.55 & 0.05 & 0.79 & 1.18 & 5.53 & 4.82 & 0.09 & 100.09 \\
\hline MI29/30 & 2 & 66.66 & 0.00 & 19.16 & 0.47 & 0.03 & 0.17 & 1.22 & 6.75 & 5.55 & 0.11 & 100.13 \\
\hline MI25/26/27 & 3 & 71.74 & 0.15 & 14.15 & 1.59 & 0.02 & 0.41 & 1.06 & 5.08 & 4.96 & 0.05 & 99.21 \\
\hline MI31/32 & 2 & 66.75 & 0.30 & 17.87 & 1.54 & 0.02 & 0.51 & 1.97 & 6.02 & 5.41 & 0.01 & 100.40 \\
\hline MI28/29 & 2 & 66.03 & 0.25 & 18.52 & 1.01 & 0.00 & 0.42 & 1.60 & 5.99 & 5.97 & 0.03 & 99.83 \\
\hline MI30 & 1 & 65.81 & 0.27 & 18.12 & 0.79 & 0.01 & 0.44 & 1.36 & 5.97 & 5.80 & 0.17 & 98.73 \\
\hline MI33 & 1 & 70.10 & 0.00 & 15.64 & 0.63 & 0.04 & 0.43 & 1.05 & 5.51 & 5.38 & 0.09 & 98.86 \\
\hline MI44/45 & 2 & 69.79 & 0.47 & 15.94 & 1.65 & 0.10 & 0.22 & 2.91 & 4.77 & 4.08 & 0.57 & 100.50 \\
\hline MI61 & 1 & 67.96 & 0.11 & 15.98 & 1.18 & 0.10 & 0.37 & 3.46 & 4.90 & 5.31 & 0.59 & 99.95 \\
\hline MI61 & 1 & 68.16 & 0.18 & 14.97 & 1.11 & 0.14 & 0.36 & 3.09 & 4.32 & 5.54 & 0.54 & 98.39 \\
\hline \multicolumn{2}{|l|}{ Average } & 68.86 & 0.44 & 16.40 & 1.39 & 0.08 & 0.43 & 1.88 & 4.96 & 4.93 & 0.23 & 99.59 \\
\hline \multicolumn{13}{|l|}{ Fe-pole } \\
\hline MI67/68 & 2 & 39.09 & 0.94 & 12.55 & 15.39 & 0.12 & 7.38 & 12.42 & 2.27 & 1.87 & 7.12 & 99.14 \\
\hline MI71/72 & 2 & 38.15 & 0.78 & 12.34 & 17.78 & 0.20 & 7.32 & 13.66 & 2.13 & 0.50 & 6.09 & 98.95 \\
\hline MI13/14 & 2 & 38.27 & 2.78 & 12.61 & 13.58 & 0.17 & 7.49 & 11.29 & 2.32 & 2.36 & 8.10 & 98.98 \\
\hline \multicolumn{2}{|l|}{ Average } & 38.51 & 1.50 & 12.50 & 15.59 & 0.16 & 7.39 & 12.46 & 2.24 & 1.58 & 7.11 & 99.02 \\
\hline
\end{tabular}

$\mathrm{n}$ is the number of microprobe analyses of the melt inclusion 
Table DR5. Composition of melt inclusions in apatite from sample at $78.1 \mathrm{~m}$

\begin{tabular}{|l|l|l|l|l|l|l|l|l|l|l|l|l|}
\hline & $\mathbf{n}$ & $\mathbf{S i O}_{2}$ & $\mathbf{T i O}_{2}$ & $\mathbf{A l}_{\mathbf{2}} \mathbf{O}_{\mathbf{3}}$ & $\mathbf{F e O}$ & $\mathbf{M n O}$ & $\mathbf{M g O}$ & $\mathbf{C a O}$ & $\mathbf{N a}_{\mathbf{2}} \mathbf{O}$ & $\mathbf{K}_{\mathbf{2}} \mathbf{O}$ & $\mathbf{P}_{\mathbf{2}} \mathbf{O}_{\mathbf{5}}$ & $\mathbf{T o t a l}$ \\
\hline Si-Pole & & & & & & & & & & & & \\
\hline MI10 & 1 & 68.31 & 0.35 & 16.35 & 1.20 & 0.00 & 0.48 & 1.29 & 4.88 & 5.78 & 0.00 & 98.64 \\
\hline MI11/12/13 & 3 & 66.75 & 1.44 & 16.17 & 2.30 & 0.05 & 0.36 & 1.02 & 4.74 & 6.34 & 0.18 & 99.35 \\
\hline MI15/16 & 2 & 67.17 & 0.93 & 16.77 & 2.09 & 0.04 & 0.40 & 1.15 & 5.07 & 5.56 & 0.16 & 99.36 \\
\hline MI20/21 & 2 & 67.62 & 0.83 & 16.62 & 1.75 & 0.00 & 0.16 & 1.40 & 5.33 & 5.54 & 0.10 & 99.35 \\
\hline MI24 & 1 & 68.34 & 0.22 & 16.69 & 1.64 & 0.04 & 0.48 & 1.22 & 4.53 & 6.49 & 0.16 & 99.81 \\
\hline MI30 & 1 & 68.63 & 0.39 & 17.11 & 0.90 & 0.00 & 0.17 & 0.85 & 4.41 & 7.26 & 0.07 & 99.79 \\
\hline MI3/4 & 2 & 69.79 & 0.06 & 17.34 & 0.62 & 0.02 & 0.08 & 1.53 & 5.93 & 5.12 & 0.09 & 100.59 \\
\hline MI3 & 1 & 70.42 & 0.00 & 16.53 & 0.32 & 0.03 & 0.11 & 0.74 & 6.34 & 4.42 & 0.12 & 99.04 \\
\hline Average & & $\mathbf{6 8 . 3 8}$ & $\mathbf{0 . 5 3}$ & $\mathbf{1 6 . 7 0}$ & $\mathbf{1 . 3 5}$ & $\mathbf{0 . 0 2}$ & $\mathbf{0 . 2 8}$ & $\mathbf{1 . 1 5}$ & $\mathbf{5 . 1 5}$ & $\mathbf{5 . 8 1}$ & $\mathbf{0 . 1 1}$ & $\mathbf{9 9 . 4 9}$ \\
\hline & & & & & & & & & & & & \\
\hline Fe-pole & & & & & & & & & & & & \\
\hline MI2 & 1 & 51.65 & 3.79 & 14.88 & 6.90 & 0.06 & 3.59 & 4.57 & 4.98 & 2.49 & 3.86 & 96.76 \\
\hline MI4 & 1 & 53.44 & 3.26 & 14.35 & 6.66 & 0.06 & 3.75 & 4.89 & 5.32 & 2.43 & 3.62 & 97.77 \\
\hline MI1/2 & 2 & 55.26 & 4.71 & 14.51 & 6.58 & 0.09 & 1.01 & 6.29 & 4.68 & 3.16 & 2.99 & 99.29 \\
\hline Average & & $\mathbf{5 3 . 4 5}$ & $\mathbf{3 . 9 2}$ & $\mathbf{1 4 . 5 8}$ & $\mathbf{6 . 7 1}$ & $\mathbf{0 . 0 7}$ & $\mathbf{2 . 7 8}$ & $\mathbf{5 . 2 5}$ & $\mathbf{4 . 9 9}$ & $\mathbf{2 . 6 9}$ & $\mathbf{3 . 4 9}$ & $\mathbf{9 7 . 9 4}$ \\
\hline
\end{tabular}

$\mathrm{n}$ is the number of microprobe analyses of the melt inclusion 